\title{
Step by step control of a deep drawing process with piezo-electric actuators in serial operation
}

\author{
Tobias Bäume ${ }^{1, a}$, Wolfgang Zorn ${ }^{2}$, Welf-Guntram Drossel ${ }^{2}$, and Gerd Rupp ${ }^{1}$ \\ ${ }^{1}$ Volkswagen AG, 38440 Wolfsburg, Germany \\ ${ }^{2}$ Fraunhofer Institute for Machine Tools and Forming Technology (IWU), 09126 Chemnitz, Germany
}

\begin{abstract}
Due to the design-driven increase in complexity of forming car body parts, it becomes more difficult to ensure a stable forming process. Piezoelectric actuators can influence the material flow of stamping parts effectively. In this article the implementation of piezoelectric actuators in a large scale sheet metal forming tool of a car manufacturer is described. Additionally, it is shown that part quality can be assessed with the help of triangulation laser sensors, which are mounted on the blankholder. The resulting flange draw-in signals were used to reduce the occurrence of wrinkling or the rate of cracking. It was shown that process control improved the quality of the stamping parts significantly.
\end{abstract}

\section{Introduction}

In sheet metal forming several parameters influence the quality of a part. If stable production conditions cannot be assured an open- or closed-loop feedback control can help to reduce rejected parts. Several feedback control approaches have been investigated to reduce the quantity of rejected parts which are caused by the adjusted process parameters.

As [1] showed it is possible to control a metal forming process if the material flow is monitored and a PID-controller varies the blank holder force. In this case a segmented blankholder is used to improve the quality of a square cup under laboratory conditions. Reference curves have been generated from sensor signals where the part quality was sufficient. Furthermore [2] designed another feedback control where master curves have been generated and unidirectional material flow sensors were recorded to monitor the process conditions. Adjustable nitrogen cylinders were controlled to regulate the blank holder force distribution of a modified wheel house liner tool [3] also used the material flow as sensor signal to control the blankholder force. Here a fuzzy control has been developed which simplifies the modelling due to a less accurate model which is adequate enough [4] examined another specially adopted tool where the active area of the blank holder is mounted on piezoelectric sensors to measure the dynamic force which is due to the sliding sheet metal between die and blankholder. The measured force signal can be converted into dynamic friction coefficients. Four embedded cavities, which vary blank holder force regions locally, are used by [5] to control a sheet metal forming process. A cascade control was designed.

\footnotetext{
${ }^{\text {a }}$ Corresponding author: tobias . baeume@volkswagen.de
}

This is an Open Access article distributed under the terms of the Creative Commons Attribution License 4.0, which permits unrestricted use, distribution, and reproduction in any medium, provided the original work is properly cited. 


\section{MATEC Web of Conferences}

The inner loop is used to minimize a linear-quadratic regulator (LQR) cost function (LQR optimal control). The outer loop reduces long term disturbances with the help of a linear learning algorithm. Due to the modified tool the concept is hardly implementable into serial production tools. For all of these concepts, a specially designed tool is needed in order to enable a local alternation of the blank holder force distribution.

\section{Set-up}

To design a process control sensors are necessary to distinguish the parameters which correlate with part quality. Several sensors for different mode of failures have been investigated in sheet metal forming. The lower boundary of the forming process can be analysed with sensors tested by [1], [6] and [7]. If the sensor signals alter from the reference signals then wrinkling will arise and the blankholder force has to be increased. The upper process limit is where necking, tearing or cracking occurs. Kergen et al. [8] shows that strain gauges are feasible to detect those mentioned failures. Beside inductive displacement sensors [9] for detecting the change of material properties the contact-less measurement of the flange draw-in via triangulation lasers are a suitable alternative for process quality evaluation [10]. The flange draw-in was identified as parameter which strongly correlates with party quality [11]. In contrast to wrinkling and crack detecting sensors flange draw-in sensors allow to estimate the part quality without exceeding the process limits. Regarding to fulfill the requirements of large scale production conditions the triangulation laser was used for further investigation. Due to the small dimensions it can be placed flexible on the outer regions of the blank holder. That allows replacing the sensor if the error pattern, which was predicted from sheet metal forming simulation, differs from reality. Finally the triangulation laser provides reliable measure signals with an accuracy of less than $0.1 \mathrm{~mm}$.

The forming tool was supplemented by five piezo actuator modules, which are used to redistribute the pressure in the flange area of the blankholder. The actuator modules corresponding power amplifiers are arranged within the tool. The energy supply and the control signals are induced via an additional interface mounted on the tool. This interface is also used for the sensor signals. The control processing was firstly realized on a rapid control prototyping system, which was arranged outside of the forming machine. This allows a convenient reconfiguration of the controller without stopping the process.

\section{Control}

\subsection{Basics}

An arbitrary process $\boldsymbol{\Psi}$, which connects an input vector $\mathbf{x}$ with an output vector $\mathbf{z}$ is defined as

$$
\mathbf{z}=\Psi(\mathbf{x})
$$

where $\mathbf{x}$ and $\mathbf{z}$ contain several single variables with the dimensions $n$ and $m$ respectively:

$$
\begin{aligned}
& \mathbf{x}=\left(x_{1}, x_{2}, \ldots, x_{n}\right) \\
& \mathbf{z}=\left(z_{1}, z_{2}, \ldots, z_{m}\right) .
\end{aligned}
$$

For each output variable $z_{i}$ a functional relation can be defined. The type of initial function mainly depends on the non-linearity of the process. If there is no information of the process, a polynomial approach should be a feasible possibility of reflecting the process. Within the scope of the presented examinations a second-order polynomial was applied:

$$
z_{i}=a_{i, 0}+a_{i, 10} \cdot x_{1}+a_{i, 20} \cdot x_{2}+\cdots+a_{i, 12} \cdot x_{1} \cdot x_{2}+a_{i, 11} \cdot x_{1}^{2}+a_{i, 22} \cdot x_{2}^{2}+\cdots
$$

The coefficients $a_{i, j}$ are calculated with a regression analysis of the results, derived from simulations or metrological examinations (see Sect. 3.2). Using Eqs. (1) and (4) an analytical description of the deep 


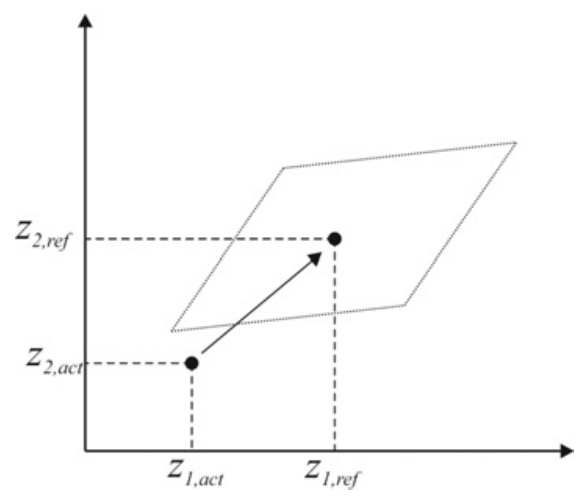

Figure 1. Target area of error-free parts.

drawing process with specified input and output parameters could be obtained. In Fig. 1 a schematic representation of the target area of error-free parts depending on the output parameters $\mathbf{z}_{\mathbf{i}}$ is given. $\mathbf{z}_{\text {act }}$ and $\mathbf{z}_{\text {ref }}$ represent the actual output values of an arbitrary production step and the given reference values respectively.

To help ensure a stable process the output values should coincidence with the reference values. Due to systematic and statistic errors there is a difference between the actual and the reference values. The reasons for this are not covered by the regression model in any case. To minimize the gap a Taylor expansion in the topical operating point $\overline{\mathrm{x}}$ can be done for $\mathbf{z}$.

$$
\begin{aligned}
& \Delta z_{1}=\left[\left.\frac{\partial z_{1}}{\partial x_{1}}\right|_{x=\bar{x}} \cdot \Delta x_{1}\right]+\left[\left.\frac{\partial z_{1}}{\partial x_{2}}\right|_{x=\bar{x}} \cdot \Delta x_{2}\right]+\cdots\left[\left.\frac{\partial z_{1}}{\partial x_{n}}\right|_{x=\bar{x}} \cdot \Delta x_{n}\right] \\
& \Delta z_{2}=\left[\left.\frac{\partial z_{2}}{\partial x_{1}}\right|_{x=\bar{x}} \cdot \Delta x_{1}\right]+\left[\left.\frac{\partial z_{2}}{\partial x_{2}}\right|_{x=\bar{x}} \cdot \Delta x_{2}\right]+\cdots\left[\left.\frac{\partial z_{2}}{\partial x_{n}}\right|_{x=\bar{x}} \cdot \Delta x_{n}\right] \\
& \vdots \\
& \Delta z_{m}=\left[\left.\frac{\partial z_{m}}{\partial x_{1}}\right|_{x=\bar{x}} \cdot \Delta x_{1}\right]+\left[\left.\frac{\partial z_{m}}{\partial x_{2}}\right|_{x=\bar{x}} \cdot \Delta x_{2}\right]+\cdots\left[\left.\frac{\partial z_{m}}{\partial x_{n}}\right|_{x=\bar{x}} \cdot \Delta x_{n}\right] .
\end{aligned}
$$

This linear system of equations can be expressed by

$$
\Delta \mathbf{z}=\mathbf{J}_{\mathbf{A}} \cdot \Delta \mathbf{x}
$$

The difference $\Delta \mathbf{z}$ between actual and reference values is known from the process, the matrix $\mathbf{J}_{\mathbf{A}}$ with its coefficients can be derived from the process model. By transforming of Eq. (6) the necessary modification $\Delta \mathbf{x}$ of the operating point can be calculated:

$$
\Delta \mathbf{x}=\mathbf{J}_{\mathbf{A}}^{-1} \cdot \Delta \mathbf{z} .
$$

In general the number of input parameters does not correlate with the number of output parameters. Thus, $\mathbf{J}_{\mathbf{A}}$ is a non-square matrix in most cases, which requires a pseudoinverse to calculate $\Delta \mathbf{x}$. This allows programming a controller, which uses the current operating point and the analytical model. The corresponding scheme of the closed loop control is shown in Fig. 2.

To illustrate this approach two scenarios were applied. In the first scenario the convergence behavior was examined by assuming a random-parametrized model with two dimensions. Due to the non-linear process model the calculated $\Delta \mathbf{z}$ does not necessarily match the output $\Delta \mathbf{z}$. This requires an additional damping factor and several steps to approach the reference point. The second scenario provides a 


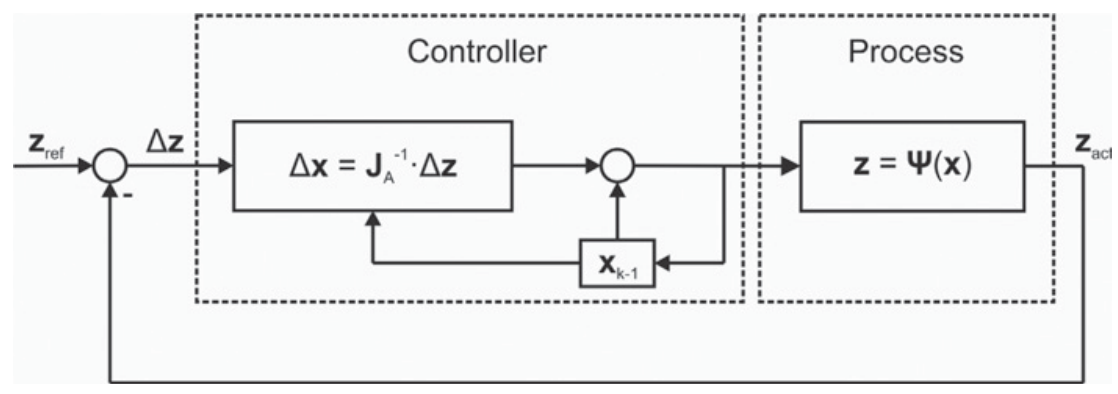

Figure 2. Control loop.

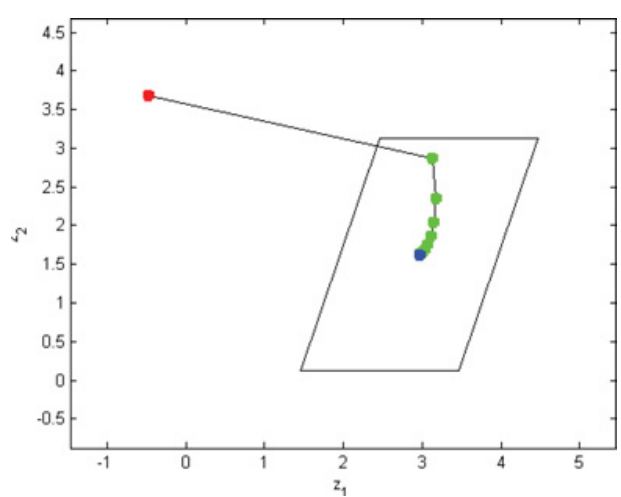

a

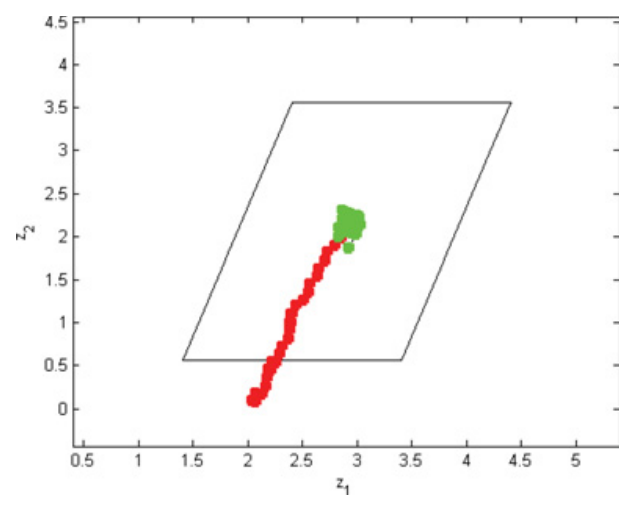

b

Figure 3. Simulation results for two scenarios (a) and (b).

disturbed process. The controller tries to keep the output variable at the stable point, while an increasing offset manipulates the process. Figure 3(a) shows corresponding simulation results for both scenarios.

\subsection{Design of experiments and process model}

As already stated the process model of the deep-drawing-process is represented by the analytic equations for the flange draw-in depending on the control parameters of the piezo actuators. Each equation satisfies the structure given in Eq. (4). To determine the parameters $a_{i, j}$ a regression analysis was used with the usage of a statistical design of experiments. As variable parameters the amplitude of the actuation voltage of each piezo actuator was chosen. To reduce the range of the design a time variant voltage was not taken into consideration. The design of experiments was achieved with computational assistance using the commercial software Cornerstone. For each of the five input parameters three categories (Low, Mean, High) were given.

The analysis of the calculated parameters $a_{i, j}$ have shown several effects of cross correlation. Thus, a single actuator does not just affect the peripheral area, but also a flange draw-in in other sectors of the blank (see Table 1). A reason for such occurrence could be the elasticity of the forming tool and the use of a die cushion, where one pressure cylinder acts on more than one barrels of the tool. This leads to a decrease of the contact pressure in the implied peripheral areas.

For evaluating the sensor signal quality regarding the suitability for a closed-loop control the parts were individually examined. For several quality conditions (without errors, necking, cracks) the range of occurred draw-in-values was considered. Figure 4 shows the corresponding box-plots for each 
ICNFT 2015

Table 1. Extract of the coefficient table.

\begin{tabular}{|c|c|c|c|}
\hline Term & Laser 1 & Laser 2 & Laser 3 \\
\hline $\mathrm{U}_{\max , \mathrm{P} 1}$ & - & 0.005858 & -0.02109 \\
\hline $\mathrm{U}_{\max , \mathrm{P} 3}$ & 0.00627 & -0.00486 & 0.00477 \\
\hline
\end{tabular}
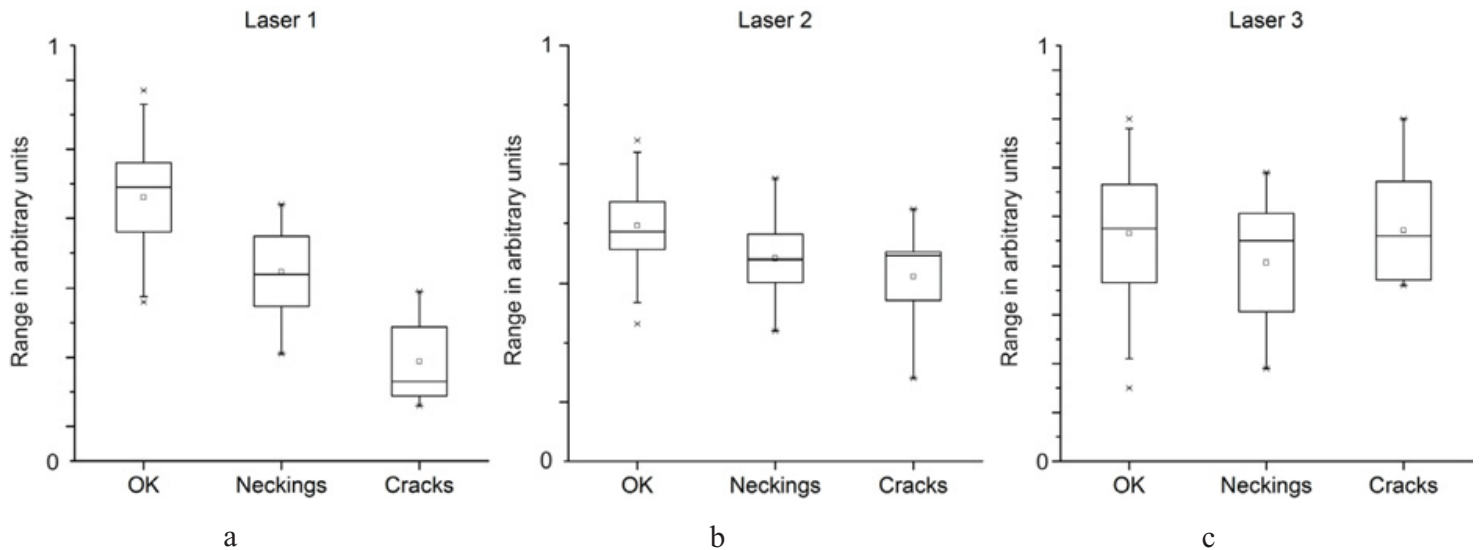

Figure 4. Box-Plot of the measured values.

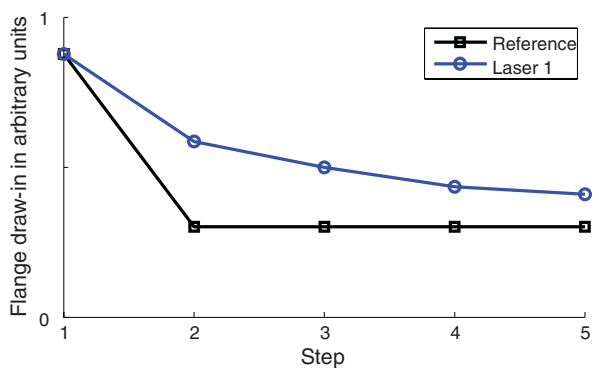

a

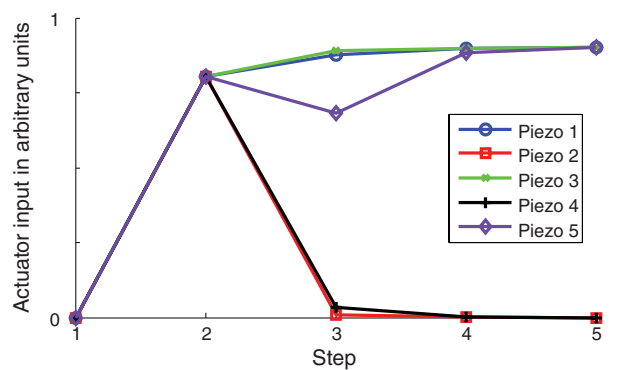

b

Figure 5. Draw-in values (a) and actuator input (b) of a step-by-step control.

laser sensor. There were overlaps of all considered quality conditions. The overlap for laser 2 and laser 3 is rather huge significant, whereas the overlap for laser 1 allows a quite good allocation of draw-in values to the part quality. Hence, only the signal of laser 1 is suitable for a closed-loop control.

\subsection{Results}

To validate the functionality of this control approach several scenarios were investigated. Due to the aforementioned reasons these scenarios only consider laser 1 as input parameter. Figure 5 shows the results for a step-by-step control of the draw-in of the blank. After an initial step the reference value was decreased. The controller calculated the new actuation forces of the five piezo actuators. Clearly visible is the step-wise convergence of the measured and actuating values. This series was intended to 

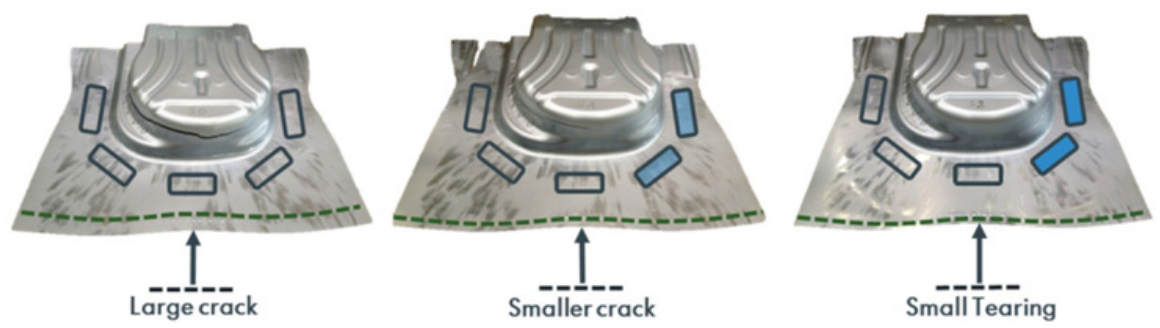

Figure 6. Results of the step-by-step control.

investigate the principle control behaviour of this approach. Assuming a larger range of operation the difference between actual value and reference value should vanish.

In another scenario the tool was adjusted to produce a large crack in the part front with no driven piezo actuators. To avoid the crack the reference value of laser 1 was increased to result in a more intensive flow of the material. As shown in Fig. 6 the controller takes three steps to minimize and eliminate the crack.

\section{Summary}

Due to the design-driven increase in complexity of forming car body parts, it becomes more difficult to ensure a stable forming process. Piezoelectric actuators can influence the material flow of stamping parts effectively by redistribute the forces in the tool. In this article the implementation of piezoelectric actuators in a large scale sheet metal forming tool of a car manufacturer was described. Additionally, it was shown that part quality can be assessed with the help of triangulation laser sensors, which are mounted on the blankholder. The resulting flange draw-in signals were used to reduce the occurrence of wrinkling or the rate of cracking. For this an analytical process model was derived from a regression analysis based on an experimental design. It was shown that process control improved the quality of the stamping parts significantly.

\section{References}

[1] B. Griesbach, Fortschritt-Berichte VDI, 547 (2000)

[2] S. Rittmeier, Systemunterstützte Umformung (2007)

[3] J.-W. Yun, Berichte aus dem IFUM, 2 (2005)

[4] M. Ziegler, Neuere Entwicklungen in der Blechumformung 427-446 (1998)

[5] B. Endelt, J. Danckert, Int J Mat Form, 3, 25-28 (2010)

[6] C. Blaich, M. Liewald, New Developments in Sheet Metal Forming, 363-384 (2008)

[7] O. Straube, Produktionstechnik, 152 (1994)

[8] R. Kergen, P. Jodogne, SAE-papers, 51-55 (1992)

[9] U. Forstmann, Berichte aus dem Produktionstechnischen Zentrum Berlin (2000)

[10] H. Bräunlich, Berichte aus dem IWU, 19 (2002)

[11] V. Thoms, M. Schatz, EFB-Forschungsbericht, 278 (2008) 